\title{
INTERAÇÕES ENTRE OS COMPORTAMENTOS DE CIDADANIA ORGANIZACIONAL, CONFIANÇA INTERPESSOAL, CONFIANÇA ORGANIZACIONAL E SUPORTE ORGANIZACIONAL
}

Data de submissão: 17/10/2017

Aceite: $31 / 07 / 2018$

Taís de Andrade ${ }^{1}$

Vania de Fátima Barros Estivalete ${ }^{2}$

Vívian Flores Costa ${ }^{3}$

Lisiane Pellini Faller ${ }^{4}$

Gabrielle Loureiro de Ávila Costa ${ }^{5}$

\section{RESUMO}

Este estudo tem como objetivo analisar as relações existentes entre os Comportamentos de Cidadania Organizacional (CCO), a Confiança Interpessoal, a Confiança Organizacional e o Suporte Organizacional, a partir da perspectiva de colaboradores do setor educacional. Para isso, realizou-se uma pesquisa de caráter descritivo do tipo survey. 0 instrumento preenchido por 988 colaboradores foi elaborado a partir da Escala de Comportamentos de Cidadania Organizacional para Trabalhadores do Conhecimento (CCO-TC), Medida de Confiança Interpessoal (CI), Escala de Confiança do Empregado na Organização (ECEO) e Escala de Percepção de Suporte Organizacional (EPSO). Os principais resultados expõem a existência de relação entre os construtos do contexto social e os CCO. A maior correlação encontrada foi entre Confiança Interpessoal e Comportamentos de Cidadania Organizacional $(r=0,462)$, sinalizando que a existência de confiança entre os indivíduos na organização é diretamente proporcional ao envolvimento destes em comportamentos positivos e discricionários em prol do bem comum.

Palavras-chave: Comportamento de Cidadania Organizacional: Confiança Interpessoal: Confiança Organizacional: Suporte Organizacional.

\footnotetext{
1 Possui graduação em Administração pela Universidade Franciscana, UFN, mestrado em Administração pela Universidade Federal de Santa Maria, UFSM e doutorado em Administração pela UFSM. Santa Maria - Rio Grande do Sul. Brasil. E-mail: tais0206@gmail.com 2 Possui graduação em Administração pela Universidade Federal de Santa Maria, UFSM, mestrado em Engenharia de Produção pela UFSM, doutorado em Agronegócios pela Universidade Federal do Rio Grande do Sul, UFRGS, e pós-doutorado em Sociologia Econômica das Organizações no SOCIUS - Centro de Investigação em Sociologia Económica e das Organizações no ISEG - Instituto Superior de Economia e Gestão da Universidade de Lisboa - Portugal. Santa Maria - Rio Grande do Sul. Brasil. E-mail: vaniaestivalete@ufsm.br 3 Possui graduação em Administração pela Universidade Federal de Santa Maria, UFSM, mestrado em Administração pela UFSM e doutorado em andamento em Administração pela UFSM. Santa Maria - Rio Grande do Sul. Brasil. E-mail: vivianfc13@gmail.com

4 Possui graduação em Desenho e Plástica pela Universidade Federal de Santa Maria, UFSM, graduação em Educação Artística pela UFSM, graduação em Administração de Empresas pela Universidade Luterana do Brasil, ULBRA, mestrado em Administração pela UFSM e doutorado em Administração pela UFSM. Santa Maria - Rio Grande do Sul. Brasil. E-mail: lisifaller@gmail.com 5 Possui graduação em Administração pela Faculdade Metodista de Santa Maria, FAMES, e mestrado em andamento em Administração pela Universidade Federal de Santa Maria. Santa Maria - Rio Grande do Sul. Brasil. E-mail: gabrielle.adm01@yahoo.com.br
} 


\section{INTRODUÇÃO}

Os Comportamentos de Cidadania Organizacional assumem grande relevância nos estudos organizacionais. Organ (1988) define a cidadania organizacional como um comportamento individual, discricionário, não explicitamente reconhecido pelo sistema formal de recompensa, que contribui para o funcionamento eficaz da organização (PODSAKOFF et al., 2014). Esses comportamentos também podem ser considerados como um conjunto de condutas interpessoais e voluntárias que sustentam o ambiente social e psicológico no qual ocorre o desempenho da tarefa (ORGAN, 1997).

Diversos estudiosos têm enfatizado a importante influência que os Comportamentos de Cidadania Organizacional (CCO) exercem sobre a viabilidade das organizações (PODSAKOFF et al., 2009). Para Yaghoubi, Yazdani e Khornegah (2011),tais comportamentos fazem com que os indivíduos assumam uma consciência e desenvolvam um comportamento pró-ativo diante de diversas situações organizacionais. Estas condutas denotam também uma preocupação com os demais indivíduos, através da manifestação de ações espontâneas e voluntárias de participação e auxílio na solução de problemas.

No entanto, no âmbito organizacional, não se pode pensar em Comportamentos de Cidadania independentes do contexto social, uma vez que o homem, como o grande construtor de todo o social, é também por ele construído (FREITAS, 2000). Ao analisar o contexto social que permeia as organizações, a Confiança Interpessoal, a Confiança Organizacional e o Suporte Organizacional contribuem sobremaneira para a compreensão acerca das interações dos indivíduos no trabalho. A confiança ocupa um lugar central nas pesquisas sobre organizações contemporâneas, uma vez que os arranjos de trabalho supõem sua existência entre os indivíduos e entre estes e a organização. Como afirma Fukuyama (1996), locais de trabalho de alta confiança caracterizam-se pelo compartilhamento de responsabilidades, senso de obrigação recíproca e menor incidência de controles rígidos.

O desenvolvimento das Ciências Organizacionais reflete a importância das relações de Confiança Interpessoal e Confiança Organizacional para a eficácia da organização, uma vez que a eficiência dentro de sistemas complexos de ação coordenada é possível quando atores confiam uns nos outros e trabalham juntos de forma eficaz (MCALLISTER, 1995). Ainda, a necessidade de confiar é tão importante quanto a satisfação de ser igualmente confiável, do contrário, não há cooperação entre as pessoas (FUKUYAMA, 1996). Sob este aspecto, a confiança, tanto em nível interpessoal quanto organizacional, pode ser considerada elemento essencial para a existência de comportamentos discricionários em benefício da coletividade.

Destaca-se ainda que as relações de confiança existentes no contexto social dependem também de outras variáveis ambientais e da experiência pessoal do indivíduo no âmbito do trabalho. A partir dessa perspectiva, o Suporte Organizacional, associado ao contexto social, emerge como um construto que pode estimular a confiança entre os membros da organização. Para Eisenberger e Stinglhamber (2011), o Suporte Organizacional está relacionado às crenças globais dos colaboradores sobre a extensão em que a organização valoriza suas contribuições e se preocupa com seu bem-estar. Assim, ao perceber Suporte Organizacional, os indivíduos tendem a confiar mais na organização, retribuindo de forma positiva, com comportamentos benéficos ao sistema social.

Desse modo, uma das motivações para a realização desse estudo recai no interesse em compreender as interações entre os construtos Confiança Interpessoal, Confiança Organizacional e Suporte da organização com os Comportamentos de Cidadania Organizacional. A análise 
conjunta destas temáticas sob a perspectiva dos trabalhadores do setor educacional enriquece o panorama sobre as interações entre os referidos construtos, uma vez que a educação contribui sobremaneira para as mudanças ocorridas na sociedade, e também vivencia o impacto das transformações sociais em sua prática (MONTEIRO, 2009). O objetivo deste trabalho consiste em analisar as relações existentes entre os Comportamentos de Cidadania Organizacional, a Confiança Interpessoal, a Confiança Organizacional e o Suporte Organizacional a partir da perspectiva de colaboradores do setor educacional.

\subsection{Comportamentos de Cidadania Organizacional}

A expressão cidadania tem sido historicamente utilizada, pelo conteúdo social, político e jurídico, para explicar a condição do ser humano como detentor de direitos e deveres enquanto membro de uma sociedade. As definições modernas de cidadania destacam que, para ser considerado um cidadão, o indivíduo precisaria pertencer a um grupo, apresentar padrões adequados de conduta e contribuir para o bem-estar da comunidade ou qualquer tipo de associação humana de forma frequente e valiosa (SMITH, 2002). Essas perspectivas foram vinculadas ao contexto organizacional e relacionadas a comportamentos do indivíduo no trabalho, permeando suas relações com outras pessoas e com a organização.

Como afirma Siqueira (1995), os Comportamentos de Cidadania Organizacional não se constituem em direitos, nem em deveres, sendo configurados muito mais como gestos de solidariedade social com o sistema, parecendo mais adequado situá-los dentro de uma visão comportamental de Civismo Organizacional. No estudo proposto por Porto e Tamayo (2003), o termo Civismo Organizacional está associado a atos espontâneos dos trabalhadores que beneficiam o sistema organizacional, que permitem formas particulares de manifestação, não prevendo retribuição formal pelo sistema organizacional. Ao analisar tal definição percebe-se similaridade ao conceito de CCO proposto por Organ (1988). Para o autor, esses comportamentos individuais e discricionários não podem nem ser impostos como obrigações as funções, nem induzidos pela garantia de uma recompensa formal. Desse modo, quando Katz e Kahn (1978) analisaram a dinâmica das organizações, constataram três tipos básicos de comportamentos fundamentais para seu funcionamento: ingresso e permanência no sistema, comportamento fidedigno (realizar os requisitos do papel de forma confiável) e comportamento inovador e espontâneo. Neste entendimento, os comportamentos inovadores e espontâneos são necessários para a organização, já que constituem um desempenho além dos requisitos de papel para a consecução das funções organizacionais. Segundo os referidos autores, estes comportamentos podem ser classificados em cinco categorias: a) atividades de cooperação com os demais membros; b) ações protetoras ao sistema; c) sugestões criativas para melhoria organizacional; d) autotreinamento para maior responsabilidade organizacional; e) criação de um clima favorável para a organização no ambiente externo.

A partir destas concepções teóricas, a noção de Comportamento de Cidadania Organizacional (CCO) foi abordada pelos estudos de Organ e seus colaboradores (BATEMAN e ORGAN, 1983; ORGAN, 1977, 1988). Por discricionário o autor explicita que o comportamento não é um requisito executável do papel ou da descrição do trabalho, e sim uma questão de escolha pessoal, de tal forma que a sua omissão não é geralmente entendida como passível de punição.

Para Eisenberger et al. (2001), ao analisar o comportamento de cidadania sob uma perspectiva de troca, a atenção foca-se no intercâmbio entre organização e indivíduo. Assim, na visão dos referidos autores, a Teoria de Troca Social explica o CCO, pois sugere que este é esperado quando o colaborador está satisfeito com a organização e se sentemotivado a responder recipro- 
camente para com esta. Desse modo, como afirmam Rapp, Bachrach e Rapp (2013), os Comportamentos de Cidadania Organizacional, impulsionados por sentimentos de reciprocidade, facilitam as interações sociais entre os colaboradores, contribuindo para a realização das tarefas de trabalho. Por outro lado, aqueles que demonstram a menor incidência desse comportamento são suscetíveis de terem poucas trocas sociais, o que pode contribuir para diminuição do desempenho nas tarefas.

Os resultados de estudos empíricos, no entanto, indicam a necessidade de teorias adicionais que poderiam explicar com maior profundidade os Comportamentos de Cidadania Organizacional (SALAMON;DEUTCH 2006). Além disso, como afirmam Dekas et al. (2013) o mundo do trabalho mudou e, fundamentalmente com esta mudança, a natureza dos Comportamentos de Cidadania Organizacional para os trabalhadores contemporâneos também foi alterada. Assim, Dekas et al (2013) propuseram uma tipologia que originou a escala de Comportamentos de Cidadania Organizacional para Trabalhadores do Conhecimento (Organizational Citizenship Behavior - Knowledge Worker), composta pelos fatores:

1. Sustentabilidade dos colaboradores: participar de atividades para manter ou meIhorar a própria saúde e bem-estar, ou para apoiar os esforços dos outros para manter a sua saúde e bem-estar.

2. Participação social: participar de atividades sociais que não são diretamente relacionadas com tarefas centrais de trabalho.

3. Virtude cívica: tomar ações indicativas de um nível macro de interesse na organização como um todo - ações que refletem um reconhecimento de ser parte de algo maior e aceitar as responsabilidades que tal adesão implica.

4. Voz: participar de atividades, fazendo sugestões, ou falando com a intenção de propor a melhoria da organização, dos produtos, ou de algum aspecto dos indivíduos, do grupo ou do funcionamento da organização.

5. Ajuda: voluntariamente ajudar os colegas de trabalho com questões relacionadas ao trabalho.

A escala de Comportamentos de Cidadania Organizacional para Trabalhadores do Conhecimento (CCO-TC) foi aplicada em inúmeras amostras e sua confiabilidade foi satisfatória, com Alphas de Cronbach entre 0,78 e 0,88 (DEKAS, 2010; DEKAS et al., 2013).

Destas categorias, três se alinham com as dimensões de cidadania preexistentes na literatura: virtude cívica, voz e ajuda. As outras duas categorias, sustentabilidade dos colaboradores e participação social, foram dimensões propostas no modelo desenvolvido por Dekas et al. (2013). Para os referidos autores, estas dimensões emergiram no contexto social relacionado aos trabalhadores do conhecimento, onde as transformações do mundo do trabalho foram significativas nas últimas décadas, demandando novo perfil de colaborador, com postura proativa e participativa na esfera social.

\subsection{Confiança Interpessoal}

A confiança interpessoal se refere à vontade de um indivíduo em estar vulnerável às ações de outra parte, baseada na expectativa de que esta realizará uma ação particularmente importante para aquele que confia, independente de sua habilidade para monitorar ou controlar a outra parte (MAYER, DAVIS; SCHOORMAN, 1995). Para Costa (2003), confiança interpessoal se refere à confiança entre indivíduos, e suas bases são as percepções pessoais ou do grupo em relação aos motivos e intenções da outra parte. 
As relações de confiança reproduzem um conjunto institucionalizado de valores, ou seja, normas que tornam estáveis e ordenam a interação social, construindo e reforçando um quadro de obrigações e expectativas compartilhadas, em que os atores sociais podem investir (REED, 2001). Para Oliveira e Tamayo (2008), ao confiar, o indivíduo acredita que o outro não agirá de modo oportunista, vindo a prejudicá-lo, sendo a confiança um indicador de que a interação social poderá ser estabelecida e mantida ao longo do tempo.

A confiança interpessoal nas organizações tem sido considerada um fenômeno complexo e dinâmico (MCCAULEY; KUHNERT, 1992; OH; PARK, 2011), sugerindo a necessidade de novos estudos que busquem ampliar a compreensão sobre este fenômeno. $O$ construto é tradicionalmente associado a consequências favoráveis, tanto para o indivíduo, quanto para a organização (ALTUNAS; BAYKAL, 2010). A confiança está sendo positivamente relacionada com o desempenho organizacional (DAVIS et al., 2000), a satisfação no trabalho (GUINOT et al., 2014), a cooperação entre os membros da equipe (LEE et al., 2011), o comprometimento organizacional (PILLAI, et al., 1999), o compromisso com as metas (SHOLIHIN et al., 2011) e o Comportamento de Cidadania Organizacional (PILLAI et al., 1999; SINGH; SRIVASTAVA, 2009).

Como afirma McAllister (1995), a comunicação aberta, a demonstração de preocupação com o outro e o oferecimento de ajuda para alcançar os objetivos são consequências típicas e observáveis de relações de confiança. Sob este aspecto, a confiança interpessoal pode funcionar também como um catalisador das interações sociais, permitindo que os membros da equipe estejam mais dispostos a oferecer ajuda e apoio um ao outro (LEE et al., 2011).

Ao constatar que a confiança interpessoal, exerce impacto sobre o desempenho das equipes, Ding e Ng (2009) sugerem uma reflexão sobre o papel dos gestores. Para os autores, os gestores de equipe possuem o papel de incentivar as interações sociais entre indivíduos e fornecer orientação sobre atitudes desejáveis em relação ao trabalho, como responsabilidade, seriedade e empreendedorismo, de tal forma que o nível de confiança interpessoal possa ser melhorado. Além disso, a confiança nos próprios gestores poderá contribuir também, para incentivar comportamentos interpessoais e voluntários que favoreçam as relações sociais (ERTÜRK, 2007).

Em relação aos elementos da confiança interpessoal, McCauley e Kuhnert (1992) e Ting (1997) indicam que a confiança interpessoal é composta pela confiança vertical e lateral. A confiança vertical se refere à relação de confiança entre um subordinado e seu superior (supervisor ou gestor) direto. Já a confiança lateral, à relação de confiança entre um colaborador e seus colegas de trabalho. Como afirma Guinot et al. (2014), estas subcategorias de confiança interpessoal sugerem que existem diferentes dimensões das relações sociais entre os trabalhadores no local de trabalho. Assim, um indivíduo pode confiar em seus colegas de trabalho, mas ter certa desconfiança em seu superior ou vice-versa (GUINOT et al., 2014).

\subsection{Confiança Organizacional}

A confiança organizacional gradualmente conquista maior importância à medida que os controles formais usados nas relações entre organizações e entre estas e seus colaboradores se tornaram insuficientes para gerar segurança às partes envolvidas BATISTA; OLIVEIRA, 2012). Como afirmam Puusa e Tolvanen (2006), a confiança é a chave para compreender a ligação entre o indivíduo e a organização e a criação de uma identidade forte com esta, além de ser responsável por fomentar comportamentos benéficos ao grupo e à própria organização. Para Li et al. (2012), a confiança organizacional se refere a percepção coletiva sobre a confiabilidade da organização. Na perspectiva de Costa (2003), a confiança na organização está associada à relação estabelecida com o sistema formal, sendo baseada em leis, regulamentos institucionais e nas práticas que a mantém. 
A ideia de confiança organizacional suscitou, portanto, um conjunto de reflexões e hipóteses sobre o papel dos processos relacionados à confiança no funcionamento das organizações (KEATING et al., 2010). Para os referidos autores, um aspecto comum a várias abordagens é a ideia de que uma organização é uma forma de assegurar a cooperação entre pessoas com interesses diferentes. Nesse sentido, quanto mais alto o grau de confiança nas organizações, maior a cooperação espontânea, sendo menor a necessidade de "aparatos legais", como sistemas de normas e regulamentos, negociados ou coercitivos, para garantir a cooperação (FUKUYAMA, 1996). Ainda, Fukuyama (1996) advoga que as organizações com nível mais elevado de confiança interna se tornam mais eficientes do ponto de vista econômico, pois não precisam investir fortemente nesses sistemas. Sob esse aspecto, Keating et al. (2010) complementam que onde não há confiança, os mecanismos de garantia, por exemplo, contratos referentes a contrapartidas a receber pela execução adequada de um trabalho, são mais complexos, mais caros e de resultados mais imprevisíveis.

Assim, para fins de investigação e diagnóstico das crenças dos colaboradores quanto à confiabilidade de sua organização de trabalho, Oliveira e Tamayo (2008) desenvolveram a Escala de Confiança do Empregado na Organização (ECEO), validada em contexto nacional. Trata-se de uma medida multidimensional composta pelas dimensões: promoção do crescimento do empregado, solidez organizacional, normas relativas à demissão dos empregados, reconhecimento financeiro organizacional e padrões éticos.

\subsection{Suporte Organizacional}

O conceito de Suporte Organizacional, introduzido nos estudos sobre comportamento organizacional a partir de 1986, por Eisenberger e seus colaboradores, traz outras perspectivas sobre as interações entre o indivíduo e a organização. Estes estudos ampliam as considerações sobre os elos que os ligam, considerando as necessidades sócio emocionais, as trocas sociais e a reciprocidade. Para Eisenberger et al. (1986), a Percepção de Suporte Organizacional (PSO) está associada à ideia de que os colaboradores desenvolvem crenças globais sobre a extensão em que a organização valoriza suas contribuições e cuida do seu bem-estar. A Teoria do Suporte Organizacional (EISENBERGER et al., 1986; EISENBERGER; STINGLHAMBER de 2011; RHOADES; EISENBERGER, 2002) considera o desenvolvimento, a natureza e as consequências de tal suporte percebido (NEVES; EISENBERGER, 2014).

De acordo com a Teoria do Suporte Organizacional, o desenvolvimento da PSO é estimulado pela tendência dos colaboradores a atribuir à organização características humanas (EISENBERGER et al., 1986). Conforme os autores, estas atribuições de características antropomórficas são consequências do comportamento dos trabalhadores ao perceber as ações dos agentes organizacionais como da própria organização. Shoss et al. (2013), argumentam ainda que a organização é moralmente e legalmente responsável pelas ações de seus membros. Atua por meio do comportamento individual de membros-chave, ocupantes de posições de gerência e de liderança (TAMAYO; TRÓCOLLI, 2002). Nesse sentido, as noções básicas que sustentam a Teoria do Suporte Organizacional incluem indicações como quanto mais os colaboradores percebem o suporte da organização, mais se comprometem com ela (SIQUEIRA; GOMIDE JR., 2008). Assim, a expectativa de reconhecimento e retribuição dada pela organização como recompensa ao trabaIho realizado se encontra diretamente relacionada à PSO, revelando a existência de expectativas de trocas sociais e reciprocidade nas interações entre indivíduo e organização. Para Chen et al. (2009), a Percepção de Suporte Organizacional atende às necessidades sócio emocionais, sendo utilizada pelos colaboradores para inferir a disposição de sua organização para recompensar seus esforços. Assim, a sensação de que a organização oferece cuidados, reconhecimento e respeito 
aos seus colaboradores pode levá-los a satisfazer necessidades socioemocionais, pois os colaboradores sentem-se membros da organização (SIQUEIRA; GOMIDE JR., 2008).

As evidências indicam que os funcionários com altos níveis de PSO julgam seu trabalho de forma mais favorável e investem mais em sua organização, contribuindo com resultados positivos nos níveis individual e organizacional (CHEN et al., 2009). No nível individual, a qualidade das trocas sociais está associada à redução do estresse, diminuição dos índices de burnout (CROPANZANO et al., 1997) e exaustão emocional (TAMAYO; TRÓCOLLI, 2002). Ainda, a PSO está relacionada positivamente ao bem-estar no trabalho (PASCHOAL et al. ,2012), à satisfação no ambiente laboral (CHEN et al., 2009; RHOADES; EISENBERG, 2002) e ao humor positivo (RHOADES; EISENBERG, 2002). Em nível organizacional, a Percepção de Suporte Organizacional poderá contribuir para o aumento do desempenho da organização, maior comprometimento afetivo dos colaboradores (CHEN et al., 2009), alcance dos objetivos e metas da equipe (KENNEDY et al., 2009). Ainda, Edwards (2009) afirma que este sentimento de gratidão irá incentivar um retorno psicológico, podendo auxiliar até mesmo com a identificação dos colaboradores com a organização, diminuição de comportamentos retaliatórios (RHOADES; EISENBERGER, 2002), absenteísmo (EISENBERGER et al., 1986) e turnover (RHOADES, EISENBERGER; AMELI, 2001).Além disso, como afirmam Neves e Eisenberger (2014), colaboradores com alta percepção de suporte organizacional podem retribuir com o aumento do desempenho do papel e também extra papel, além de demonstrar comportamentos éticos no trabalho (CHEN et al., 2009; TREMBLAY; LANDREVILLE, 2014).

\section{MÉTODO}

Este estudo descritivo foi guiado pelo método survey, o qual, segundo Fowler Jr. (2013), visa produzir estatísticas quantitativas ou descrições numéricas sobre aspectos de uma população estudada.

A população-alvo desta pesquisa foi composta de trabalhadores do setor educacional privado, pertencentes a cinco cidades do estado do Rio Grande do Sul. No presente estudo foram aplicados 1510 questionários, obtendo-se um retorno de 65\%, os quais representam 988 indivíduos, pertencentes a treze organizações de ensino privadas, localizadas nas cidades de Santa Maria/RS, Passo Fundo/RS, Porto Alegre/RS, Cruz Alta/RS e Caxias do Sul/RS. O questionário foi elaborado a partir dos seguintes instrumentos:

(a) Escala de Comportamentos de Cidadania Organizacional para Trabalhadores do Conhecimento (CCO-TC), desenvolvido por Dekas (2010) e Dekas et al. (2013) composto por 23 variáveis e cinco fatores (Sustentabilidade dos colaboradores, Participação social, Virtude cívica, Voz e Ajuda), possuindo uma escala tipo likert de 5 pontos, variando de (1) discordo totalmente a 5 (concordo totalmente). A Escala CCO-TC foi traduzida e validada no Brasil por Andrade (2017). Após a realização da Análise Fatorial Confirmatória, a escala CCO-TC teve o número de fatores alterados, sendo composta por: Virtude cívica, Voz, Altruísmo aos colegas e Ajuda (ANDRADE, 2017), analisados no presente estudo.

(b) Medida de Confiança Interpessoal, incluindo a dimensão Confiança nos colegas de Trabalho, proposta por Oh e Park (2011), composta por 4 variáveis e Confiança no superior, proposta por Nyhan (2000), possuindo também 4 variáveis. A referida medida possui uma escala tipo likert de 5 pontos, variando de (1) discordo totalmente a (5) concordo totalmente. A Medida de Confiança Interpessoal foi traduzida para o português e validada no Brasil por Andrade (2017).

(c) Escala de Confiança do Empregado na Organização (ECEO), desenvolvida por Oliveira e Tamayo (2008), em sua versão reduzida, composta por 28 questões e cinco fatores 
(Promoção do crescimento do empregado, Solidez organizacional, Normas quanto à demissão, Reconhecimento financeiro organizacional e Padrões éticos), possuindo uma escala tipo likert de 5 pontos, variando de (1) discordo totalmente a (5)concordo totalmente. No estudo desenvolvido por Andrade (2017), após a realização da Análise Fatorial Confirmatória, foi excluído o fator Normas à demissão, o qual não apresentou valores satisfatórios em relação à confiabilidade. (ANDRADE, 2017). Assim, este fator foi excluído da presente pesquisa.

(d) Escala de Percepção de Suporte Organizacional (EPSO), desenvolvida por Eisenberger et al. (1986) e validada no contexto nacional por Siqueira (1995), composta por 6 variáveis, possuindo uma escala tipo likert de 5 pontos variando de (1) discordo totalmente a (5) concordo totalmente.

Para facilitar a compreensão, apresenta-se o desenho da pesquisa conforme a Figura 1.

Figura 1: Desenho de pesquisa

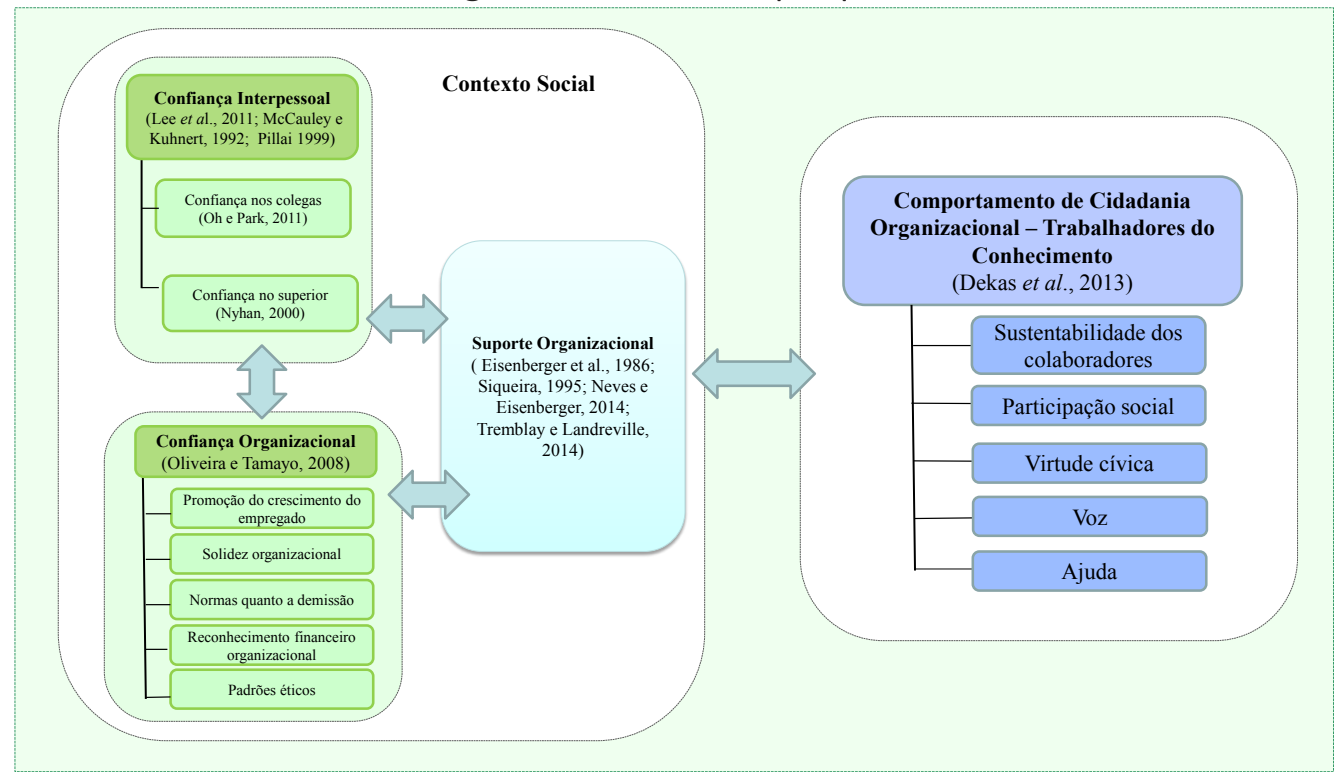

Fonte: Elaborado pelos autores

Os dados obtidos nesta etapa do estudo foram analisados com o apoio do software SPSS (Statistical Package for the Social Sciences). Como os dados da presente pesquisa configuraram-se como paramétricos, foi realizado o cálculo do Coeficiente de Correlação de Pearson, o qual indica a força de associação entre quaisquer duas variáveis (HAIR et al., 2009).

\section{RESULTADOS}

O perfil da amostra caracteriza-se por $28,1 \%$ de homens e $71,9 \%$ de mulheres, possuindo, em média, 35,54 anos de idade (desvio padrão 0,98), sendo que destes, 52,3\% têm de 30 a 44 anos. Em relação ao estado civil, a maioria dos respondentes é representada por indivíduos casados $(43,3 \%$ ) e $38,3 \%$ por indivíduos solteiros.

Quanto à escolaridade dos participantes da pesquisa, houve o predomínio de indivíduos com pós-graduação completa $(39,85 \%)$ e com ensino superior completo $(22,8 \%)$. Constatou- 
-se também que grande parte dos respondentes possui o curso superior em Pedagogia $(25,5 \%)$ e Administração (14,5\%). Na amostra investigada houve o predomínio dos cargos de professor $(47,3 \%)$ e auxiliar administrativo (13,2\%), sendo que, em média, os respondentes possuem 5,02 anos de tempo de empresa (desvio padrão 1,08), 4,35 anos de tempo no cargo (desvio padrão $0,99)$ e exercem suas atividades em 8 h diárias.

\subsection{Relações entre os Comportamentos de Cidadania Organizacional, Confiança Interpessoal, Confiança Organizacional e Suporte Organizacional.}

A fim de estabelecer as relações entre os Comportamentos de Cidadania Organizacional, a Confiança Interpessoal, a Confiança Organizacional e o Suporte Organizacional, calculou-se o coeficiente de Correlação de Pearson, cujos resultados são apresentados na Tabela 1. De acordo com Hair et al. (2009), o coeficiente de correlação de Pearson mensura a associação linear entre duas variáveis métricas e varia de $-1,00 \mathrm{a}+1,00$. Quanto maior o coeficiente de correlação, mais forte a ligação ou o nível de associação entre as variáveis, sendo que este pode ser positivo ou negativo (HAIR et al., 2009. As variações do coeficiente de correlação entre 0,01 e 0,2 são consideradas associações muito baixas; entre 0,2 e 0,39 são classificadas como baixas; entre 0,4 e 0,69 são designadas moderadas; entre 0,7 e 0,89 são consideradas associações altas; e entre 0,9 e 1 são classificadas como muito altas (PESTANA; GAGEIRO, 2003).

Tabela 1: Matriz de correlação entre os fatores dos Comportamentos de Cidadania Organizacional, Confiança Interpessoal, Confiança Organizacional e Suporte Organizacional

\begin{tabular}{|c|c|c|c|c|c|c|c|c|c|c|}
\hline Corr. & Voz & $\begin{array}{l}\text { Altr. } \\
\text { col }\end{array}$ & Ajuda & $\begin{array}{c}\text { Conf. } \\
\text { col }\end{array}$ & $\begin{array}{l}\text { Conf. } \\
\text { sup }\end{array}$ & $\begin{array}{c}\text { Pro. } \\
\text { cresc. }\end{array}$ & $\begin{array}{l}\text { Soli. } \\
\text { org. }\end{array}$ & $\begin{array}{l}\text { Rec. } \\
\text { fin }\end{array}$ & $\begin{array}{l}\text { Padr. } \\
\text { eti }\end{array}$ & $\begin{array}{l}\text { Sup. } \\
\text { Org }\end{array}$ \\
\hline Virt.civ. & , 448 &, $344^{* *}$ & ,311 ** & $\underset{* *}{252}$ & ,356 ** & ,384 ${ }^{* *}$ & ,311** & ,308 ** & ,387 &, $378^{* *}$ \\
\hline Voz & & , $355^{* *}$ &, $419^{* *}$ & , 187 &, $244^{* *}$ &, $187^{* *}$ & ,269 ** & ,107 ** &, $282^{* *}$ & $\underset{* *}{248}$ \\
\hline Altr.col & & & ,489** &, $376^{* *}$ & ,317 ** & ,260 ** & , 249 ** & ,219 ** & ,370 ** & $\begin{array}{c}304 \\
* *\end{array}$ \\
\hline Ajuda & & & & $\begin{array}{c}352 \\
* *\end{array}$ & ,314 ** & 239 ** & ,293 ** & ,183 ** & ,331 ** & $\begin{array}{c}279 \\
* *\end{array}$ \\
\hline $\begin{array}{c}\text { Conf. } \\
\text { col }\end{array}$ & & & & &, $527^{* *}$ & ,320** & ,385 ** & ,292 ** & ,469 ** & ,478 \\
\hline $\begin{array}{l}\text { Conf. } \\
\text { sup }\end{array}$ & & & & & &, $460^{* *}$ & ,409 ** & ,432 ** &, $526^{* *}$ &, $560^{* *}$ \\
\hline $\begin{array}{l}\text { Pro. } \\
\text { cresc. }\end{array}$ & & & & & & &, $527^{* *}$ &, $703^{* *}$ &, $593^{* *}$ & ,662 \\
\hline $\begin{array}{c}\text { Sol. } \\
\text { orga. }\end{array}$ & & & & & & & &, $536^{* *}$ & ,700 ** &, $582^{* *}$ \\
\hline Rec.fin & & & & & & & & &, $521^{* *}$ & , 626 \\
\hline $\begin{array}{l}\text { Padr. } \\
\text { eti }\end{array}$ & & & & & & & & & &, $687^{* *}$ \\
\hline
\end{tabular}


** Correlações significantes ao nível de 0,01

Virt. Civ. - Virtude Cívica; Altr. Col.- Altruísmo aos Colegas; Conf. Col - Confiança nos Colegas; Conf. Sup. - Confiança nos Superiores; Pro. Cresc- Promoção do Crescimento dos Empregados; Sol. Orga.- Solidez Organizacional; Rec. Fin. - Reconhecimento Financeiro Organizacional; Padr. Eti. - Padrões Éticos; Sup. Org. - Suporte Organizacional

Fonte: Dados da pesquisa

As interações entre os fatores dos Comportamentos de Cidadania Organizacional para Trabalhadores do Conhecimento e a Confiança Interpessoal, revelaram a maior associação entre as dimensões Confiança nos colegas e Altruísmo aos colegas, sendo esta considerada positiva e baixa $(r=0,376)$. Assim, estes resultados remetem à compreensão de que, para os trabalhadores do setor educacional investigado, quanto maior a confiança nos colegas de trabalho, mais evidentes serão os comportamentos altruístas em relação aos colegas.

Para McAllister (1995), a confiança interpessoal está alicerçada em bases cognitivas e afetivas, representando a extensão em que uma pessoa está segura e disposta a agir em relação à outra. A confiança interpessoal sinaliza que o indivíduo acredita que o outro não irá agir de modo oportunista, podendo a confiança perdurar por um longo tempo (OLIVEIRA; TAMAYO, 2008). Desta forma, quanto maior a confiança nos colegas, maior será a frequência do auxílio voluntário a eles com questões relacionadas às atividades laborais (DEKAS et al., 2013). Ainda, a confiança nos colegas pode estar associada à norma da reciprocidade, como defendem os autores Reed (2001) e McAllister (1995), em que um indivíduo agirá de certa forma esperando que outros irão agir de forma semelhante no futuro.

Quanto às relações entre os fatores da Confiança Organizacional e os Comportamentos de Cidadania Organizacional para Trabalhadores do Conhecimento, a maior correlação foi constatada entre Padrões éticos e Virtude cívica, considerada positiva e baixa $(r=0,387)$. Sob este aspecto, pode-se constatar que quanto mais o trabalhador perceber os princípios éticos da organização, como a honestidade, igualdade, transparência, responsabilidade, compromisso e respeito (OLIVEIRA; TAMAYO, 2008), maior será o seu envolvimento com ações indicativas de um nível macro de interesse da organização, que refletem o reconhecimento de ser parte de um todo maior (DEKAS et al., 2013).

Em relação às correlações entre Suporte Organizacional e o CCO-TC, constatou-se que a maior correlação foi observada entre os fatores Suporte Organizacional e Virtude Cívica $(r=0,378)$, a qual configura-se como positiva e baixa. Deste modo, quanto mais os trabalhadores percebem a preocupação da organização com seu bem-estar e a valorização de suas contribuições (PAILLE et al., 2000; DULAC et al., 2008), maior será o seu envolvimento em comportamentos voluntários, relacionados a participação espontânea em eventos e atividades promovidas pela organização em prol de seu desenvolvimento (DEKAS et al., 2013; DEKAS, 2010).

Já em relação às interações entre a Confiança Interpessoal e a Confiança Organizacional, a maior correlação foi observada entre os fatores Confiança nos superiores e Padrões éticos, sendo esta positiva e moderada $(r=0,526)$. Para os trabalhadores do setor educacional investigado, quanto maior a confiança nos superiores maior será a confiança nos padrões éticos da organização, sugerindo a importância do papel dos gestores em relação à confiança dos indivíduos na organização. Este fato pode estar associado ao que Levinson (1965) define como a personificação da organização, a qual pode ocorrer por meio dos agentes que exercem poder sobre cada um dos colaboradores. Assim, ao confiar em seus superiores, os trabalhadores tendem a confiar mais na organização em que trabalham. 
Ainda, ao analisar os fatores entre a Confiança Interpessoal e o Suporte Organizacional, pode-se observar que a maior correlação existente foi entre a Confiança nos superiores e o Suporte Organizacional $(r=0,560)$, a qual foi positiva e moderada. Da mesma forma, quanto mais os indivíduos confiam em seus superiores, maior será sua percepção em relação ao suporte oferecido pela organização. A Teoria do Suporte Organizacional defende que o desenvolvimento da PSO está associado à tendência dos colaboradores em atribuir à organização características humanas, principalmente ao associar as ações dos agentes organizacionais como ações da própria organização (EISENBERGER et al., 1986). As interações entre Confiança nos superiores e Suporte Organizacional podem estar alicerçadas nesta perspectiva teórica.

Por fim, a maior correlação entre os fatores da Confiança Organizacional e o Suporte Organizacional foi observada entre Padrões Éticos e Suporte Organizacional $(r=0,687)$, sendo esta positiva e moderada. Esse resultado permite inferir que quanto mais os trabalhadores percebem o apoio por parte da organização, maior será sua avaliação em relação à honestidade, confiabilidade e ética empresarial. Como destacam Dulac et al. (2008) e Paille, Bourdeau e Galois (2010) a confiança em uma organização tende a aumentar quando os trabalhadores percebem que esta mostra preocupação com o seu bem-estar e valoriza suas contribuições.

\section{CONSIDERAÇÕES FINAIS}

No presente estudo, buscou-se analisar as relações existentes entre os Comportamentos de Cidadania Organizacional, a Confiança Interpessoal, a Confiança Organizacional e o Suporte Organizacional, a partir da perspectiva de colaboradores do setor educacional. Com base em uma pesquisa survey, realizada por meio de questionários, uma primeira constatação relevante refere-se ao alto nível de escolaridade dos participantes, sendo que a maioria possui formação em nível de pós-graduação e ensino superior completo. Os altos níveis de escolaridade dos indivíduos da amostra podem estar associados às exigências da Lei de Diretrizes e Bases da Educação Nacional, número 9394, de 20 de dezembro de 1996, a qual especifica a formação profissional em cursos de graduação para docentes e profissionais de educação para administração, planejamento, inspeção, supervisão e orientação educacional para a educação básica.

Considerando a relação entre os construtos investigados, percebeu-se que as associações foram baixas, moderadas, altas. Ao avaliar as correlações entre os construtos do contexto social e os CCO, a maior correlação foi encontrada entre Virtude Cívica e Padrões Éticos ( $r=$ $0,387)$. Tal correlação sinaliza que ao perceber os princípios éticos da organização, os trabalhadores tendem a apresentar maior participação nas atividades organizacionais, bem como maior envolvimento com os interesses da empresa. Ainda pode-se depreender que existe associação positiva e moderada entre os demais construtos do contexto social (Confiança Organizacional e Suporte Organizacional) e os CCO.

Do ponto de vista pragmático, esses achados sinalizaram para a importância das variáveis do contexto social, presentes na organização, e sua interação com os CCO. Desse modo, as organizações podem desenvolver estratégias e ações que promovam o desenvolvimento da Confiança Interpessoal e Organizacional e do Suporte Organizacional, fomentando o reconhecimento, a colaboração e a interação entre seus membros. Ressalta-se, ainda, que devem ser considerados os limites do estudo. O primeiro recai sobre sua abrangência, uma vez que a amostra utilizada se caracterizou por ser não probabilística por conveniência, sendo os resultados e conclusões obtidos restritos à realidade estudada. Assim, para realizar generalizações acerca do setor educacional, é importante a ampliação da amostra de indivíduos e organizações investigadas. 
Outro ponto que pode ser levantado como limitação, refere-se à realização de uma pesquisa exclusivamente quantitativa, sendo recomendado utilizar em estudos posteriores, metodologias multimétodos, visando aprofundar os resultados obtidos. Ainda, ressalta-se como limitação do estudo a discussão dos dados exclusivamente a partir da análise de Correlação, sugerindo-se para estudos futuros maior aprofundamento dos dados, incluindo também a análise de regressão múltipla.

\section{REFERÊNCIAS BIBLIOGRÁFICAS}

ALTUNAS, S.; BAYKAL, U. Relationship between nurses' organizational trust levels and their organizational citizenship behaviours. Journal of Nursing Scholarship, v. 42, n. 2, p. 186-194, 2010.

ANDRADE, S. M.; FISCHER, A. L.; STEFANO, S. R. Confiança organizacional e interpessoal como uma dimensão de clima organizacional. BASE - Revista de Administração e Contabilidade da UNISINOS, v. 12, n. 2, p. 155-166, 2015.

BATEMAN, T. S.; ORGAN, D. W. Job satisfaction and the good soldier: the relationship between affect and employee "citizenship". Academy of Management Journal, v. 26, n. 4, p. 587-595, 1983.

BATISTA, R. L.; OLIVEIRA, A. F. Antecedentes da confiança do empregado na organização. Estudos de Psicologia, v. 17, n. 2, p. 247-254, 2012.

BRASIL. Lei no 9.394/96, de 20 de dezembro de 1996. Lei de diretrizes e bases da educação nacional. Disponível em: http://www.planalto.gov.br/ccivil_03/Leis/L9394.htm.

CHEN, Z. et al. Perceived organizational support and extra-role performance: which leads to which? Journal of Social Psychology, v. 149, n. 1, p. 119-124, 2009.

CROPANZANO, R. et al. The relationship of organizational politics and support to work behaviors, attitudes, and stress. Journal of Organizational Behavior, v. 18, n. 2, p. 159-180, 1997.

COSTA, A. C. Work team trust and effectiveness. Personnel Review, v. 32, n. 5, p. 605-623, 2003.

DAVIS, J. H. et al. The trusted general manager and business unit performance: empirical evidence of a competitive advantage. Strategic Management Journal, v. 21, n. 5, p. 563576, 2000.

DING, Z.; NG, F. Personal construct-based factors affecting interpersonal trust in a project design team. Journal of Construction Engineering and Management, v. 136, n. 2, p. 227-234, 2009.

DEKAS, K. H. Citizenship in context: investigating the effects of work group climate on organizational citizenship perceptions and behavior. 2010. 287 f. Tese (Doutorado em Administração) -University of Michigan, Ann Arbor, Michigan, 2010. 
DEKAS, K. H. et al. Organizational citizenship behavior, version 2.0: a review and qualitative investigation of OCBs for knowledge workers at Google and beyond. The Academy of Management Perspectives, v. 27, n. 3, p. 219-237, 2013.

DULAC, T. et al. Not all responses to breach are the same: the interconnection of social exchange and psychological contract processes in organizations. Academy of Management Journal, v. 51, n. 6, p. 1079-1098, 2008.

EDWARDS, M. R. HR, perceived organisational support and organisational identification: an analysis after organisational formation. Human Resource Management Journal, v. 19, n. 1, p. 91-115, 2009.

EISENBERGER, R., HUNTINGTON, R.; SOWA, D. Perceived organizational support. Journal of Applied Psychology, v. 71, n. 3, p. 500-507, 1986.

EISENBERGER, R. et al. Reciprocation of perceived organizational support. Journal of Applied Psychology, v. 86, n. 51, p. 42-51, 2001.

EISENBERGER, R.; STINGLHAMBER, F. Perceived organizational support: fostering enthusiastic and productive employees. Washington: American Psychological Association, 2011.

ERTÜRK, A. Increasing organizational citizenship behaviors of Turkish academicians: mediating role of trust in supervisor on the relationship between organizational justice and citizenship behaviors. Journal of Managerial Psychology, v. 22, n. 3, p. 257-270, 2007.

FOWLER JR, F. J. Survey research methods. Sage publications, 2013.

FREITAS, M. E. Contexto social e imaginário organizacional moderno. Revista de Administração de Empresas, v. 40, n. 2, p. 6-15, 2000.

FUKUYAMA, F. Confiança: as virtudes sociais e a criação da prosperidade. Rio de Janeiro: Rocco, 1996.

GUINOT, J., CHIVA, R.; ROCA-PUIG, V. Interpersonal trust, stress and satisfaction at work: an empirical study. Personnel Review, v. 43, n. 1, p. 96-115, 2014.

HAIR, J. F. et al. Análise multivariada de dados. 6. ed. Porto Alegre: Bookman, 2009.

KATZ, D.; KAHN, R. L. Psicologia social das organizações. São Paulo: Atlas., 1978

KEATING, J., SILVA, I.; VELOSO, A. L. M. Confiança organizacional: teste de um modelo. Braga: Associação Portuguesa de Psicologia, 2010.

KENNEDY, F. A. et al. Effects of organizational support on potency in work teams the mediating role of team processes. Small Group Research, v. 40, n. 1, p. 72-93, 2009.

LEE, D.; STAJKOVIC, A. D.; CHO, B. Interpersonal trust and emotion as antecedents of cooperation: evidence from Korea1. Journal of Applied Social Psychology, v. 41, n. 7, p. 1603-1631, 2011. 
LEVINSON, H. Reciprocation: the relationship between man and organization. Administrative Science Quarterly, v. 9, n. 4, p. 370-390, 1965.

LI, P. P.; BAI, Y.; XI, Y. The contextual antecedents of organizational trust: a multidimensional crosslevel analysis. Management and Organization Review, v. 8, n. 2, p. 371-396, 2012.

MCALLISTER, D. J. Affect-and cognition-based trust as foundations for interpersonal cooperation in organizations. Academy of management journal, v. 38, n. 1, p. 24-59, 1995.

MCCAULEY, D. P.; KUHNERT, K. W. A theoretical review and empirical investigation of employee trust in management. Public Administration Quarterly, v. 16, n. 2, p. 265-284, 1992.

MAYER, R. C.; DAVIS, J. H.; SCHOORMAN, F. D. An integrative model of organizational trust. Academy of management review, v. 20, n. 3, p. 709-734, 1995.

MONTEIRO, M. I. N. L. Educação: novos cenários e perspectivas. Revista Tempos e Espaços em Educação, v. 2, p. 107-118, 2009.

NEVES, P.; EISENBERGER, R. Perceived organizational support and risk taking. Journal of Managerial Psychology, v. 29, n. 2, p.187-215, 2014.

NYHAN, R. C. Changing the paradigm trust and its role in public sector organizations. The American Review of Public Administration, v. 30, n.1, p. 87-109, 2000.

$\mathrm{OH}, \mathrm{Y}$. O.; PARK, J. J. New link between administrative reforms and job attitude: the role of interpersonal trust in peers as a mediator on organizational commitment. International Review of Public Adminsitration, v. 16, n. 3, p. 65-88, 2011.

OLIVEIRA, A. F.; TAMAYO, A. Confiança do empregado na organização. In: SIQUEIRA, M. M. M. (Org.). Medidas do comportamento organizacional: ferramentas de diagnóstico e gestão. Porto Alegre: Artmed, 2008.

ORGAN, D. W. Organizational citizenship behavior: the good soldier syndrome. Canada: Lexington Books, 1988.

ORGAN, D. W. Organizational citizenship behavior: its construct clean-up time. Human Performance, v. 10, p. 85-97, 1997.

PAILLÉ, P., BOURDEAU, L.; GALOIS, I. Support, trust, satisfaction, intent to leave and citizenship at organizational level: a social exchange approach. International Journal of Organizational Analysis, v. 18, n. 1, p. 41-58, 2010.

PASCHOAL, T.; TORRES, C. V.; PORTO, J. B. Felicidade no trabalho: relações com suporte organizacional e suporte social. Revista de Administração Contemporânea, v. 14, n. 6, p. 1054-1072, 2010.

PESTANA, M. H.; GAGEIRO, J. Análise de dados para Ciências Sociais: a complementaridade do SPSS. 5 ed. Lisboa: Edições Sílabo, 2008. 
PILLAI, R; SCHRIESCHEIM, C. A.; WILLIAMS, E. S. Fairness perceptions and trust as mediators for transformational and transactional leadership: a two-sample study. Journal of Management, $v$. 25, n. 6, p. 897-933, 1999.

PODSAKOFF, N. P. et al. Consequences of unit-level organizational citizenship behaviors: A review and recommendations for future research. Journal of Organizational Behavior, v. 35, n. S1, p. S87-S119, 2014.

PODSAKOFF, N. P. et al. Individual and organizational-level consequences of organizational citizenship behaviors: a meta-analysis. Journal of Applied Psychology, v. 94, n. 1, p. 122-141, 2009.

PORTO, J. B.; TAMAYO, A. Desenvolvimento e validação da escala de civismo nas organizações. Estudos de psicologia, v. 8, n. 3, p. 393-402, 2003.

PUUSA, A.; TOLVANEN, U. Organizational identity and trust. Electronic Journal of Business Ethics and Organization Studies, v. 11, n. 2, p. 29-33, 2006.

RAPP, A. A.; BACHRACH, D. G.; RAPP, T. L. The influence of time management skill on the curvilinear relationship between organizational citizenship behavior and task performance. Journal of Applied Psychology, v. 98, n. 4, p. 668-677, 2013.

REED, M. I. Organization, trust and control: a realist analysis. Organization studies, v. 22, n. 2, p. 201-228, 2001.

RHOADES, L.; EISENBERGER, R. Perceived organizational support: a review of the literature. Journal of Applied Psychology, v. 87, n. 4, p. 698-714, 2002.

RHOADES, L.; EISENBERGER, R.; ARMELI, S. Affective commitment to the organization: the contribution of perceived organizational support. Journal of Applied Psychology, v. 86, n. 5, p. 825-836, 2001.

SALAMON, S. D.; DEUTSCH, Y. OCB as a handicap: an evolutionary psychological perspective. Journal of Organizational Behavior, v. 27, n. 2, p. 185-199, 2006.

SINGH, U.; SRIVASTAVA, K. B. L. Interpersonal trust and organizational citizenship behavior. Psychological Studies, v. 54, n. 1, p. 65-76, 2009.

SIQUEIRA, M. M. M.; GOMIDE JR, S. Suporte no trabalho. In: SIQUEIRA, M. M. M. (Org.) Medidas do comportamento organizacional: ferramentas de diagnóstico e de gestão. Porto Alegre: Artmed, 2008.

SIQUEIRA, M. M. M. Antecedentes de comportamentos de cidadania organizacional: a análise de um modelo pós-cognitivo. Tese de Doutorado. Instituto de Psicologia, Universidade de Brasília, Brasília, 1995.

SHOLIHIN, M. et al. Goal-setting participation and goal commitment: Examining the mediating roles of procedural fairness and interpersonal trust in a UK financial services organisation. The British Accounting Review, v. 43, n. 2, p.135-146, 2011. 
SHOSS, M. K. et al. Blaming the organization for abusive supervision: the roles of perceived organizational support and supervisor's organizational embodiment. Journal of Applied Psychology, v. 98, n. 1, p. $158-162,2013$.

SMITH, R. Modern citizenship. In: ISIN, F. E.; TURNER, B. (Eds.) Handbook of Citizenship, London: Sage, 2002.

TAMAYO, M. R.; TRÓCCOLI, B. T. Exaustão emocional: relações com a percepção de suporte organizacional e com as estratégias de coping no trabalho. Estudos de Psicologia, v. 7, n. 1, p. 37-46, 2002.

TING, Y. Determinants of job satisfaction of federal government employees. Personnel Administration, v. 26, n. 3, p. 313-334, 1997.

TREMBLAY, M.; LANDREVILLE, P. E. Information sharing and citizenship behaviors mediating the roles of empowerment, procedural justice, and perceived organizational support. International Journal of Business Communication, v. 52, n. 4, p. 347-368, 2014.

YAGHOUBI, N. M.; YAZDANI, B. O.; KHORNEGAH, K. The relationship between Organizational Citizenship Behavior (OCB) and Social Capital (SC). American Journal of Scientific Research, v. 24, p. 121-126, 2011. 